\title{
Vaccins rétroviraux : approche comparative en médecine humaine et vétérinaire
}

La mise au point d'un vaccin contre le SIDA constitue l'un des grands défis de la fin de ce siècle $[1,2]$. Pourtant, après dix années de recherche, les perspectives de succès semblent toujours lointaines.

Les rétrovirus affectent aussi bien les animaux que les humains, et de nombreuses recherches portent sur la prévention des maladies qu'entraînent ces virus dans le bétail ou chez les animaux de compagnie. La confrontation des résultats obtenus sur les rétrovirus humains et animaux ne pourrait-elle aider à résoudre des problèmes communs, à imaginer des solutions ou à ouvrir de nouvelles voies de recherche [3] ? D'où la proposition de l'un d'entre nous (G. de Thé) à la Fondation Mérieux de réunir les meilleurs experts des vaccins rétroviraux. Ces scientifiques se sont retrouvés les 23 et 24 juin dernier, au Centre de conférences des Pensières, au bord du lac d'Annecy, pour une réunion «sans frontière entre les deux médecines, humaine et vétérinaire».

Un vaccin, oui, mais comment?

«Il n'existe pas d'alternative aux vaccins contre le VIH», affirmait Luc Montagnier (Unité d'oncologie virale, Institut Pasteur, Paris) en préambule aux discussions, «même s'il existe des approches complémentaires pour empêcher la dissémination de l'infection virale (modification des comportements sexuels, protection contre les maladies sexuellement transmissibles, prévention de l'hyperactivation du système immunitaire, traitements antiviraux...) ».
Les débats se sont articulés autour de cinq grandes questions, soulevées au cours de la table ronde initiale:

- Est-il possible de vacciner efficacement contre des rétrovirus?

- Le seul objectif est-il de protéger contre l'infection primaire ou bien faut-il tenter de protéger contre la maladie?

- Quels types de vaccins sont capables d'induire la meilleure protection?

- Quelles sont les bases immunologiques de la protection?

- La variabilité virale représente-t-elle un obstacle incontournable à la mise au point d'un vaccin contre le VIH?

Peut-on vacciner contre des rétrovirus?

L'immunoprophylaxie des infections rétrovirales est possible: la preuve en est fournie par la médecine vétérinaire. Des vaccins contre le FeLV (Feline Leukemia Virus) sont déjà commercialisés. Edward Hoover (Colorado State University, Fort Collins, CO, USA) a rappelé que les premiers essais s'étaient toutefois révélés difficiles: dans certaines circonstances, l'immunisation avec un FeLV inactivé ou avec des sous-unités virales augmentait la sensibilité à l'infection [4]. Mais les chats peuvent être protégés efficacement contre l'infection avec un canarypox exprimant les gènes gag et env du FeLV [5], ou avec un vaccin entier inactivé $[4,6]$ et contre la maladie avec des protéines purifiées $(\mathrm{Gp} 70)$ associées à des adjuvants (immunostimulating complex ou Iscom) $[7,8]$ ou avec des protéines recombinantes [9] (Edward Hoover, USA;
Oswald Jarrett, université de Glasgow, Royaume-Uni).

«Mais il est difficile de transposer les résultats obtenus avec le FeLV (un oncorétrovirus) au VIH (un lentirétrovirus) », soulignait O. Jarrett. «Ces deux types de virus possèdent des propriétés différentes." Le FeLV se multiplie relativement lentement et son génome est relativement stable. Cette caractéristique distingue les oncorétrovirus des lentirétrovirus (voir Tableau I et Encadré). Les lentivirus, depuis ceux des petits ruminants jusqu'au VIH, accumulent les variations génomiques, ainsi que l'a exposé Jean-François Mornex (Inra et École nationale vétérinaire de Lyon). «En 100000 ans d'infection dans le monde, le HTLV (human T-cell leukemia virus) a moins varié que le VIH en dix ans d'incubation chez un seul individu infecté » précisait Robin Weiss (Chester Beatty Laboratories, Londres).

Autre différence importante: l'infection lentirétrovirale naturelle conduit toujours à une infection persistante (Tableau II). C'est ce que l'on observe aussi bien pour les infections à virus Maedi-visna chez les ovins (Ian McConnell, université de Cambridge, Royaume-Uni) que pour les infections à EIAV (equine infectious anemia virus) chez les chevaux (Ronald C. Montelaro, university of Pittsburgh School of Medicine, PA, USA), ou pour les infections par le VIH chez les humains. En revanche, le système immunitaire peut parfois éliminer les oncorétrovirus, notamment le FeLV.

Cependant, bien que les chevaux infectés par l'EIAV le restent à vie, ils 


\begin{tabular}{|c|c|c|c|}
\hline \multicolumn{4}{|c|}{$\begin{array}{c}\text { Tableau I } \\
\text { ONCORÉTROVIRUS }\end{array}$} \\
\hline Virus & Espèce hôte & Cellules/organes cibles & Proliférations tumorales \\
\hline MuLV (Murine leukemia Virus) & Murins & Lymphocytes/Thymus & Lymphomes \\
\hline FeLV (Feline leukemia virus) & Félins & Lymphocytes T/Thymus & $\begin{array}{l}\text { Lymphosarcomes, lymphomes, } \\
\text { immunodépression }\end{array}$ \\
\hline BLV (Bovine leukemia virus) & Bovins, ovins & Lymphocytes B/Thymus & Leucémies, lymphomes \\
\hline $\begin{array}{l}\text { SRV (Simian retrovirus) } \\
\text { (rétrovirus de type D) }\end{array}$ & Simiens & Lymphocytes T4 & $\begin{array}{l}\text { Sarcomes, carcinomes } \\
\text { et immunodépression }\end{array}$ \\
\hline $\begin{array}{l}\text { HTLV-1/HTLV-2 (Human T- } \\
\text { cell leukemia virus) }\end{array}$ & Humains & Lymphocytes T4/Thymus? & $\begin{array}{l}\text { Leucémies, maladies } \\
\text { dégénératives du système } \\
\text { nerveux (paraparésie spastique } \\
\text { tropicale) }\end{array}$ \\
\hline
\end{tabular}

Tableau II

CARACTÉRISTIQUES PATHOGÉNIQUES DES LENTIRÉTROVIRUS

\begin{tabular}{|c|c|c|c|}
\hline Virus & Animal hôte & Cellules cibles/organes cibles & Maladie \\
\hline Maedi-visna & Ovins & $\begin{array}{l}\text { Macrophages, monocytes } \\
\text { Poumon, SNC }\end{array}$ & $\begin{array}{l}\text { Pneumonies interstitielles, } \\
\text { leucoencéphalomyélites } \\
\text { démyélinisantes progressives fatales }\end{array}$ \\
\hline $\begin{array}{l}\text { CAEV (virus de } \\
\text { I'arthrite/encéphalite } \\
\text { caprine) }\end{array}$ & Caprins & $\begin{array}{l}\text { Macrophages } \\
\text { Membranes synoviales, SNC }\end{array}$ & Arthrite, encéphalites \\
\hline $\begin{array}{l}\text { EIAV (virus } \\
\text { de I'anémie } \\
\text { infectieuse équine) }\end{array}$ & Équins & Macrophages, monocytes & $\begin{array}{l}\text { Fièvre, diarrhées, anémie, } \\
\text { thrombocytopénies régressives }\end{array}$ \\
\hline $\begin{array}{l}\text { SIV (virus de } \\
\text { l'immunodéficience } \\
\text { simienne) }\end{array}$ & Simiens & $\begin{array}{l}\text { Cellules T4, macrophages, } \\
\text { monocytes }\end{array}$ & Déficit immunitaire \\
\hline $\begin{array}{l}\text { VIH (virus de } \\
\text { l'immunodéficience } \\
\text { humaine) }\end{array}$ & Humains & $\begin{array}{l}\text { Cellules T4, macrophages, } \\
\text { monocytes, cellules de Langerhans }\end{array}$ & Déficit immunitaire \\
\hline
\end{tabular}


Les rétrovirus infectieux sont à l'origine de maladies variées chez les animaux et chez l'homme: proliférations malignes du système hématopoïétique et conjonctif, immunodépressions, maladies auto-immunes, maladies neurodégénératives.

On classe généralement ces virus en deux grandes familles*: les rétrovirus oncogènes lou oncorétrovirus) et les lentivirus.

- Les rétrovirus oncogènes lou oncorétrovirus) sont associés à différents types de leucémies, de lymphomes, de sarcomes, d'épithéliomas, dans différentes espèces animales (Tableau I) et leur étude a mené à la découverte des oncogènes. Chez les humains, on a identifié un seul rétrovirus pathogène: le HTLV (human T-cell leukemia virus). Le HTLV-1 est responsable de leucémies $T$ de l'adulte, répandues dans le sud du Japon et dans les Caraïbes [15, 16]. Ce même virus est également à l'origine d'un syndrome neurodégénératif, la paraparésie spastique tropicale, et de maladies auto-immunes (uvéites, arthrites, myosites, polymyosites). Le HTLV-2, isolé initialement chez un patient atteint de leucémie à tricholeucocytes, a été associé de façon sporadique à des symptômes neurologiques et hématologiques. Ce virus se propage chez les toxicomanes par voie intraveineuse.

- Les lentirétrovirus sont responsables de maladies dégénératives concernant le système immunitaire (déficits immunitaires, maladies auto-immunes) et le système nerveux central. Ils sont à l'origine de maladies pulmonaires et de maladies osseuses et articulaires chez les animaux. Chez l'homme, le VIH1 et le $\mathrm{VIH}-2$ sont à l'origine de l'épidémie mondiale de SIDA.

* Une autre classe de rétrovirus, les virus spumeux, n'a été associée à aucune maladie, même chez des hôtes immunodéprimés, bien que ces virus exercent un effet cytopathique sur les cellules en culture.

$\mathrm{m} / \mathrm{s} n^{\circ} 1$, vol. 12 , janvier 96 peuvent rester indéfiniment asymptomatiques. Les animaux sont alors remarquablement résistants à de nouvelles expositions au virus. Mais la maladie peut toutefois réapparaître à l'occasion d'un stress. Le système immunitaire est donc capable de protéger, sinon contre l'infection, du moins contre la maladie.

\section{Protéger contre l'infection ou contre la maladie?}

Pourra-t-on, par la vaccination, faire plus que la nature et protéger contre les infections lentivirales? C'est l'objectif que s'étaient initialement fixé les chercheurs pour le vaccin contre le SIDA... Mais d'après les résultats obtenus et d'après l'étude des modèles vétérinaires, il semble plus réaliste de rechercher une protection contre la maladie, sans empêcher totalement l'infection... à condition de pouvoir résoudre les problèmes de sécurité.

\section{Quels types de vaccins?}

De nombreuses approches vaccinales ont été testées. Divers vaccins prototypes ont permis d'obtenir une protection, soit contre l'infection, soit contre la maladie (Tableaux III et IV). Le groupe de Janice Clements (Johns Hopkins University School of Medicine, Baltimore, MD, USA) étudie, dans un modèle de mouton transgénique, le rôle de la glycoprotéine d'enveloppe dans la pathogénie du virus visna, ainsi que les possibilités de protection avec cet antigène.

\section{Des résultats variables}

Les réponses à la vaccination sont variables selon les espèces et selon le type de vaccin utilisé. Ainsi, le vaccin vaccine-Env du BLV (bovine leukemia virus) entraîne une protection chez les ovins (espèce sensible) mais pas chez les bovins, hôtes naturels du virus $[10,11]$ (A. Burny, université de Bruxelles, Belgique, Y. Ikawa, université de Tokyo, Japon). On n'a pas observé de protection contre le virus de l'immunodéficience féline (FIV) avec des protéines recombinantes produites dans E. coli ou dans des cellules d'insecte (O.Jarrett), probable- ment du fait de l'absence de glycosylation correcte. De même, l'injection d'ADN codant pour plusieurs protéines de SIVmac (le virus de l'immunodéficience simienne du macaque) n'a permis de prévenir ni l'infection, ni la maladie, en dépit de l'induction d'une réponse CTL contre Env et Gag. (Harriett L. Robinson, University of Massachusetts Medical Center, Worcester, MA, USA).

Si l'injection du virus recombinant exprimant la protéine d'enveloppe du HTLV-1 est suivie de rappels effectués avec la glycoprotéine, la protection des lapins est abolie (G. Franchini, National Cancer Institute, Bethesda, USA). On a obtenu un vaccin efficace contre le FeLV avec un virus canarypox recombinant [5], alors qu'avec d'autres virus vecteurs (vaccine, virus herpès), aucun effet protecteur n'a été observé [12] (E. Hoover, O. Jarrett).

Les résultats obtenus dans les recherches vétérinaires vont dans le même sens que les recherches dans les modèles expérimentaux du SIDA: les virus vivants atténués semblent les plus aptes à protéger les animaux contre la maladie (Tableaux III et IV). Mais le problème de la sécurité de tels vaccins n'est pas encore résolu. S'il protège contre l'infection par le virus sauvage, le virus CAEV (virus de l'arthrite-encéphalite caprine) délété du gène tat conserve une certaine pathogénicité (R. Vigne Inserm, Marseille). Les virus délétés peuvent, à la suite de recombinaisons, retrouver leur pouvoir de multiplication (James Stott, National Institute for Biological Standard and Control, Potters Barr, Royaume-Uni). Les virus SIV (simian immunodeficiency virus) atténués par délétion des gènes nef et/ou vpr sont inoffensifs et protecteurs chez les macaques adultes [13], mais ils induisent la maladie lorsqu'ils sont administrés aux macaques nouveau-nés, comme l'a démontré Ruth Ruprecht (m/s $n^{\circ} 7$, vol. 11, p. 1052) [14] (Dana Farber Institute and Harvard Medical School, Boston, MA, USA). Ces observations l'ont conduite à introduire le concept de seuil de réplication virale et à proposer une nouvelle définition de l'atténuation virale. "En effet, expliquait R. Ruprecht, la pathogénicité rétrovirale ne devient apparente 
Tableau III

VACCINS ANTI-ONCORÉTROVIRAUX

\begin{tabular}{|c|c|c|c|c|c|}
\hline $\begin{array}{l}\text { Approche } \\
\text { vaccinale }\end{array}$ & MuLV & FeLV & BLV & SRV & HTLV \\
\hline $\begin{array}{l}\text { Virus entier } \\
\text { inactivé }\end{array}$ & & $\begin{array}{l}\text { Protection contre } \\
\text { l'infection et la maladie } \\
\text { (mais risque } \\
\text { de sensibilisation) }\end{array}$ & & $\begin{array}{l}\text { Protection } \\
\text { contre I'infection }\end{array}$ & \\
\hline $\begin{array}{l}\text { Virus vivant } \\
\text { atténué }\end{array}$ & Protection & & & & \\
\hline $\begin{array}{l}\text { Vecteur } \\
\text { recombinant } \\
\text { exprimant } \\
\text { des gènes } \\
\text { rétroviraux }\end{array}$ & $\begin{array}{l}\text { Vaccine-Env: } \\
\text { protection } \\
\text { partielle } \\
\text { contre } \\
\text { la maladie }\end{array}$ & $\begin{array}{l}\text { Canarypox-Gag, Env: } \\
\text { protection contre } \\
\text { l'infection } \\
\text { Herpes-Gap, Env: pas } \\
\text { de protection } \\
\text { Vaccine gp } 85 \text { Env: } \\
\text { pas de protection }\end{array}$ & $\begin{array}{l}\text { Vaccine-Env gp } 51 \\
\text { et/ou gp } 30: \\
\text { Ovins: protection } \\
\text { contre I'infection } \\
\text { Bovins: pas } \\
\text { de protection }\end{array}$ & $\begin{array}{l}\text { Vaccine-Env gp } 70 \\
\text { et gp } 22: \text { protection } \\
\text { contre I'infection }\end{array}$ & $\begin{array}{l}\text { ALVAC-Env gp 53: } \\
\text { protection partielle } \\
\text { contre I'infection } \\
\text { chez le lapin } \\
\text { ALVAC-Env gp } 53+ \\
\text { rappels de gp 53: } \\
\text { pas de protection }\end{array}$ \\
\hline $\begin{array}{l}\text { Sous-unités } \\
\text { virales } \\
\text { purifiées }\end{array}$ & & $\begin{array}{l}\text { Gp 70-ISCOM: } \\
\text { protection contre } \\
\text { la maladie } \\
\text { (Certains cas: Gp } 70 \text { ou } \\
\text { Gp } 85: \text { pas de protection } \\
\text { ou sensibilisation) }\end{array}$ & $\begin{array}{l}\text { Ovins: gp } 51 \\
\text { de BLV+ adjuvant: } \\
\text { protection } \\
\text { contre l'infection }\end{array}$ & & \\
\hline $\begin{array}{l}\text { Sous-unités } \\
\text { virales } \\
\text { recombinantes }\end{array}$ & & $\begin{array}{l}\text { rgp: protection } \\
\text { ou absence } \\
\text { de protection }\end{array}$ & & & \\
\hline $\begin{array}{l}\text { Corrélats } \\
\text { de l'immunité }\end{array}$ & $\begin{array}{l}\text { Immunité } \\
\text { cellulaire }\end{array}$ & $\begin{array}{l}\text { Anticorps } \\
\text { neutralisants? }\end{array}$ & $\begin{array}{l}\text { Anticorps } \\
\text { neutralisants? }\end{array}$ & $\begin{array}{l}\text { Anticorps } \\
\text { neutralisants }\end{array}$ & \\
\hline
\end{tabular}

MuLV: murine leukemia virus; FeLV: feline leukemia virus; $B L V$ : bovine leukemia virus ; SRV: simian retrovirus; $H T L V:$ human T cell leukemia virus; ALVAC: vaccin avec un virus canarypox atténué (société Virogenetics); rgp: glycoprotéines recombinantes; ISCOM: immunostimulating complex.

que lorsque le taux de réplication virale dépasse un seuil donné. En dessous de ce seuil la réplication n'entraîne pas de maladie, même si le virus est totalement pathogène. En revanche, un virus faiblement réplicatif peut dépasser ce seuil chez des sujets permissifs (nouveau-nés, sujets immunodéprimés).»

\section{Protection ou sensibilisation?}

La vaccination contre des rétrovirus peut même, dans certaines circonstances, faciliter l'apparition de la maladie lors d'un contact ultérieur rappelé que certains essais de vaccination contre le FeLV, avec un virus inactivé ou avec des glycoprotéines virales, avaient abouti à une augmentation de la sensibilité à l'infection. Chez le chat, l'infectivité du FIV sauvage a été augmentée après vaccination avec un vecteur vaccine recombinant exprimant la protéine d'enveloppe du FIV (Albert DME Osterhaus, Université Erasmus, Rotterdam, Pays-Bas). La vaccination de poneys avec une protéine recombinante (gp 90) d'EIAV favorise plutôt qu'elle n'inhibe l'infection (R.C. Montelaro). Des chèvres vaccinées avec un CAEV inactivé développent une maladie plus sévère que les animaux non immunisés (Kalman Perk, université de Jérusalem, Israël).

\section{Quels corrélats de l'immunité?}

Il est difficile de prévoir l'effet de la vaccination, en raison de l'absence de corrélats clairs de l'immunité. La protection naturelle contre le FeLV est liée à la présence d'anticorps neutralisants. Pourtant, les vaccins ayant prouvé leur efficacité n'induisent pas ou peu de ces anticorps. Dans d'autres cas, la vaccination induit une forte réponse anticorps, sans entraîner de protection. 
Tableau IV

VACCINS ANTILENTIRÉTROVIRUS

\begin{tabular}{|c|c|c|c|c|c|c|}
\hline $\begin{array}{l}\text { Approche } \\
\text { vaccinale }\end{array}$ & CAEV & MAEDI-VISNA & EIAV & FIV & SIV & VIH \\
\hline $\begin{array}{l}\text { Virus entier } \\
\text { inactivé }\end{array}$ & $\begin{array}{l}\text { Maladie } \\
\text { plus sévère }\end{array}$ & $\begin{array}{l}\text { OPPV : pas } \\
\text { de protection } \\
\text { contre } \\
\text { l'infection }\end{array}$ & $\begin{array}{l}\text { Virus homologue: } \\
100 \% \text { de protection } \\
\text { contre l'infection } \\
\text { Virus hétérologue: } \\
\text { protection contre } \\
\text { la maladie }\end{array}$ & $\begin{array}{l}\text { Protection } \\
\text { contre I'infection }\end{array}$ & $\begin{array}{l}\text { Protection contre } \\
\text { la maladie à VIS } \\
\text { avec VIH-2 inactivé }\end{array}$ & $\begin{array}{l}\text { VIH-2 (chez le singe) } \\
\text { Protection contre } \\
\text { l'infection par virus } \\
\text { homologue } \\
\text { Protection contre } \\
\text { la maladie par virus } \\
\text { hétérologue }\end{array}$ \\
\hline $\begin{array}{l}\text { Virus vivant } \\
\text { atténué }\end{array}$ & $\begin{array}{l}\text { Mutation de vif: } \\
\text { pas de protection } \\
\\
\text { Délétion de tat: } \\
\text { protection partielle } \\
\text { contre l'infection, } \\
\text { pathogénicité } \\
\text { résiduelle }\end{array}$ & & $\begin{array}{l}\text { Protection contre } \\
\text { la maladie }\end{array}$ & & $\begin{array}{l}\text { VISmac1A11: } \\
\text { protection contre } \\
\text { la maladie } \\
\text { Délétion de nef et de } \\
\text { vpr: protection chez } \\
\text { les adultes } \\
\text { Induction de la maladie } \\
\text { par le vaccin chez les } \\
\text { nouveau-nés }\end{array}$ & \\
\hline $\begin{array}{l}\text { Virus } \\
\text { recombinant }\end{array}$ & $\begin{array}{l}\text { Vaccine-gp de env: } \\
\text { pas de protection }\end{array}$ & & & $\begin{array}{l}\text { Vaccine } \\
\text { recombi- } \\
\text { nante-Env-: } \\
\text { pas de protection } \\
\text { Maladie } \\
\text { plus sévère }\end{array}$ & $\begin{array}{l}\text { Vaccine-gp60 } \\
\text { protection } \\
\text { Vaccine-Nef: } \\
\text { protection partielle } \\
\text { contre la maladie } \\
\text { Virus Semliki Forest } \\
\text { recombinant-Env: } \\
\text { pas de protection } \\
\text { contre l'infection; } \\
\text { protection partielle } \\
\text { contre la maladie }\end{array}$ & $\begin{array}{l}\text { NYVAC-Gag, Pol, } \\
\text { Env de VIH-1: } \\
\text { protection contre } \\
\text { VIH-2 chez } \\
\text { le macaque Rhésus }\end{array}$ \\
\hline $\begin{array}{l}\text { Sous-unités } \\
\text { virales } \\
\text { purifiées }\end{array}$ & & & $\begin{array}{l}\text { Protection contre } \\
\text { l'infection par virus } \\
\text { homologue } \\
50 \% \text { de protection } \\
\text { contre la maladie } \\
\text { avec virus } \\
\text { hétérologue }\end{array}$ & $\begin{array}{l}\text { Protéines Env: } \\
\text { protection } \\
\text { partielle contre } \\
\text { l'infection } \\
\text { (diminution de la } \\
\text { charge virale) }\end{array}$ & $\begin{array}{l}\text { + adjuvant: protection } \\
\text { partielle contre } \\
\text { la maladie }\end{array}$ & \\
\hline $\begin{array}{l}\text { Sous-unités } \\
\text { virales } \\
\text { recombinantes }\end{array}$ & & & $\begin{array}{l}\text { rgp } 90: \text { pas } \\
\text { de protection } \\
\text { contre virus } \\
\text { homologue ou } \\
\text { hétérologue } \\
\text { Facilitation }\end{array}$ & $\begin{array}{l}\text { Protéines rEnv } \\
\text { (produites dans } \\
\text { E. coli ou } \\
\text { baculovirus) pas } \\
\text { de protection } \\
\text { contre I'infection } \\
\text { Env V3: pas } \\
\text { de protection } \\
\text { (ou augmentation) }\end{array}$ & & $\begin{array}{l}\text { Canarypox-VIH-2 } \\
\text { (singe) protection } \\
\text { contre l'infection } \\
\text { (homologue) ou la } \\
\text { maladie } \\
\text { (hétérologue) }\end{array}$ \\
\hline $\begin{array}{l}\text { ADN viral } \\
\text { plasmidique }\end{array}$ & $\begin{array}{l}\text { ADN proviral } \\
\text { avec délétion de vif: } \\
\text { pas de protection } \\
\text { Pas d'Ac }\end{array}$ & & & & $\begin{array}{l}\text { Gènes codant pour } \\
\text { Gag p24, Env: } \\
\text { diminution de la } \\
\text { charge virale, pas de } \\
\text { protection contre } \\
\text { la maladie }\end{array}$ & \\
\hline $\begin{array}{l}\text { Corrélats } \\
\text { d'immunité }\end{array}$ & $\begin{array}{l}\text { Absence de } \\
\text { protection en } \\
\text { présence d'Ac }\end{array}$ & $\begin{array}{l}\text { Absence de } \\
\text { protection en } \\
\text { présence d'Ac } \\
\text { neutralisants }\end{array}$ & $\begin{array}{l}\text { Lente maturation } \\
\text { de la réponse } \\
\text { humorale } \\
\text { et cellulaire } \\
\text { Ac non nécessaire }\end{array}$ & $\begin{array}{l}\text { Anticorps } \\
\text { neutralisants }\end{array}$ & & \\
\hline
\end{tabular}

CAEV: virus de l'arthrite/encéphalite caprine; EIAV: virus de l'anémie infectieuse équine; FIV: virus de l'immunodéficience féline; SIV: virus de l'immunodéficience simienne; VIH: virus de l'immunodéficience humaine; OPPV: ovine progressive pneumonia virus (virus de la famille du virus maedi-visna). NYVAC: vaccin par vecteur poxvirus atténué (société Virogenetics).

$m / s n^{\circ} 1$, vol. 12 , janvier 96 
Selon R. Montelaro, les réponses protectrices suscitées par les vaccins entiers, vivants ou atténués, sont liées à l'induction d'anticorps de grande avidité, spécifiques des déterminants conformationnels, présentés par les glycoprotéines d'enveloppe du virus sous forme native. Les anticorps facilitants réagissent peu, en revanche, avec les protéines d'enveloppe natives, et reconnaissent essentiellement les déterminants antigéniques linéaires de ces protéines d'enveloppe. D'après les résultats observés dans la leucémie bovine, l'immunité humorale pourrait jouer un rôle majeur dans l'infection primaire, mais les anticorps seraient incapables d'éliminer le génome viral intégré dans l'ADN de l'hôte, sans immunité cellulaire (Y. Ikawa). Mais l'immunisation de singes macaques avec des lipopeptides correspondant aux épitopes $\mathrm{T}$ et avec des peptides antigéniques correspondant aux épitopes neutralisants du SIV n'ont pas permis de protéger les animaux contre l'infection, en dépit d'une réponse anamnestique (R. Kurth, Paul Ehrlich Institut, Langen, Allemagne). S'il a été démontré que les anticorps neutralisants jouent un rôle majeur dans la protection des chimpanzés contre l'infection à VIH-1, une protection croisée contre le VIH-2 a été obtenue chez le macaque Rhésus en dépit de l'absence totale d'anticorps neutralisants contre le VIH-2 (Marc Girard, Institut Pasteur, Paris). Il est probable cependant, selon $\mathrm{M}$. Girard, qu'il soit nécessaire d'obtenir à la fois une forte réponse en anticorps neutralisants et une forte réponse $\mathrm{T}$ cytotoxique pour avoir un vaccin réellement efficace.

\section{Est-il possible de vacciner contre des virus hétérologues?}

Faudra-t-il incorporer, dans les vaccins contre le VIH, des antigènes des différents sous-types qui infectent les différents groupes à risque? En effet, comme l'a exposé Max Essex (Harvard AIDS Institute, Boston, MA, USA), trois grands sous-types de VIH-1 infectent la population: le sous-type B se trouve essentiellement dans les pays occidentaux tandis que le VIH-C est
E est en train de supplanter le soustype C. Ces différents sous-types semblent adaptés aux différentes voies de transmission: hétérosexuelle pour les sous-types $\mathrm{C}$ et $\mathrm{E}$, qui infectent en priorité les cellules de Langerhans (nombreuses au niveau du vagin, du col de l'utérus et du pénis ); voie homosexuelle et voie sanguine pour le sous-type B qui infecte préférentiellement les lymphocytes sanguins.

Mais on peut espérer protéger contre des virus hétérologues: selon les données présentées par G. Biberfeld (Karolinska Institute, Stockholm, Suède), l'injection de VIH-2 protège les singes cynomolgus contre la maladie causée par des virus hétérologues (SIVmac). Il pourrait en être de même chez l'homme. Max Essex a rappelé que l'infection par le VIH-2 entraînait un certain degré de protection contre le VIH-1 et Marc Girard a indiqué que la vaccination de singes Rhésus avec un vecteur recombinant contenant les gènes codant pour les protéines Gag, Pol et Env du VIH-1, suivie de l'injection de protéines d'enveloppe, entraînait une protection contre le VIH-2. Ainsi, il ne serait peut-être pas nécessaire de vacciner avec une souche virale étroitement liée à celles qui se propagent sur le terrain.

\section{Des vaccins thérapeutiques?}

Est-il possible, dans une première étape, d'envisager un vaccin thérapeutique, d'action plus immédiate et plus facile à tester en clinique? Mais leur formulation pourrait être différente de celle des vaccins prophylactiques, soulignait Stanley Plotkin (PasteurMérieux-Connaught). C'est ce qu'illustraient les résultats des études préliminaires présentés par A. Burny. Ces essais ont été mis en œuvre après la constatation qu'une augmentation des taux plasmatiques d'IFN- $\alpha$ précède l'apparition du SIDA. Des sujets séropositifs ont été vaccinés contre cette cytokine (avec un IFN- $\alpha$ émulsifié avec un adjuvant). Leur évolution s'est avérée plus favorable que celle des sujets témoins. Mais il est encore trop tôt pour que l'on puisse tirer des conclusions définitives de ces observations, réalisées sur un nombre limité de patients
Les résultats et discussions de cette réunion seront publiés très prochainement dans leur intégralité dans un numéro spécial de la revue AIDS and Human Retroviruses.

\section{RÉFÉRENCES}

1. Girard M. Vaccins contre le SIDA: espoirs et réalités. médecine/sciences $1989 ; 5$ : 152-8.

2. Lévy JP. Le problème d'un vaccin contre le SIDA. médecine/sciences 1995; 11 : 407-19.

3. Schlienger K, Mancini M, Tiollais P, Michel M. Vaccination contre le SIDA: évaluation chez les primates. médecine/sciences 1995 ; 11 : 985-93.

4. Pedersen NC, Johnson L, Birch O, et al. Possible immunoenhancement of persistent viremia by feline leukemia virus envelope glycoprotein vaccines in challenge-exposure situations where whole inactivated virus vaccines were protective. Vet Immunol Immunopathol 1986; $11: 123-8$.

5. Tartaglia J, Jarrett O, Neil JC, Desmettre P, Paoletti E. Protection of cats against feline leukemia virus by vaccination with a canarypox recombinant ALVAC-FL. $J$ Virol 1993; 67: 2370-5.

6. Hoover EA, Perigo NA, Quackenbush SL, et al. Protection against feline leukemia virus infection by use of an inactivated virus vaccine. J Am Vet Med Assoc 1991; 199 : 1392405.

7. Osterhaus A, Weijer K, UytdeHaag F, et $a l$. Induction of protective immune response in cats by vaccination with feline leukemia virus ISCOM. J Immunol 1985 ; 135 : 5916.

8. Osterhaus A, Weijer K, UytdeHaag F, et al. Serological responses in cats vaccinated with FeLV ISCOM and an inactivated FeLV vaccine. Vaccine 1989; 7 : 137-41.

9. Marciani DJ, Kensil CR, Beltz GA, et al. Genetically engineered subunit vaccine against feline leukemia virus: Protective immune response in cats. Vaccine 1991 ; 9: 89-96.

10. Portetelle D, Limbach K, Burny A, et al. Recombinant vaccinia virus expression of the bovine leukemia envelope gene and protection of immunized sheep against infection. Vaccine 1991 ; 9 : 194-200.

11. Onuma M, Hodatsu T, Yamamoto S, et al. Protection by vaccination against bovine leukemia virus infection in sheep. Am J Vet Res 1984; 48 : 117-25.

12. Gilbert JH, Pedersen NC, Nunberg JH. Feline leukemia virus envelope protein expression encoded by a recombinant vaccinia virus: Apparent lack of immunogenicity in vaccinated animals. Virus Res 1987; 7: 4967.

$m / s n^{\circ} 1$, vol. 12 , janvier 96 
13. Gibbs JS, Regier DA, Desrosiers RC. Construction and in vitro properties of SIV mutants with deletions in nonessential genes. AlDS Res Hum Retroviruses 1994; 10 : $607-16$.

14. Baba TW, Jeong YS, Penninck D, Bronson R, Greene MF, Ruprecht RM. Pathogenicity of live attenuated SIV after mucosal infection of neonatal macaques. Science $1995 ; 267: 1820-5$.

15. De Thé G, Gessain A. HTLV-1 1992, aspects moléculaires et immuno-prévention. médecine/sciences 1992; 8: 205-7.
16. Koralnik I, Gessain A. Virus HTLV-I: structure et fonction des protéines de la région $\mathrm{pX}$. médecine/sciences 1994; 10 : 296-305.

TIRÉS À PART

B. Dodet.

\section{Betty Dodet}

Fondation Mérieux, 17, rue Bourgelat, BP 2021, 69227 Lyon Cedex 02, France.

Guy De Thé

Unité d'épidémiologie des virus oncogènes, département des rétrovirus, Institut Pasteur, 25, rue du Docteur-Roux, 75724 Paris Cedex 15, France.

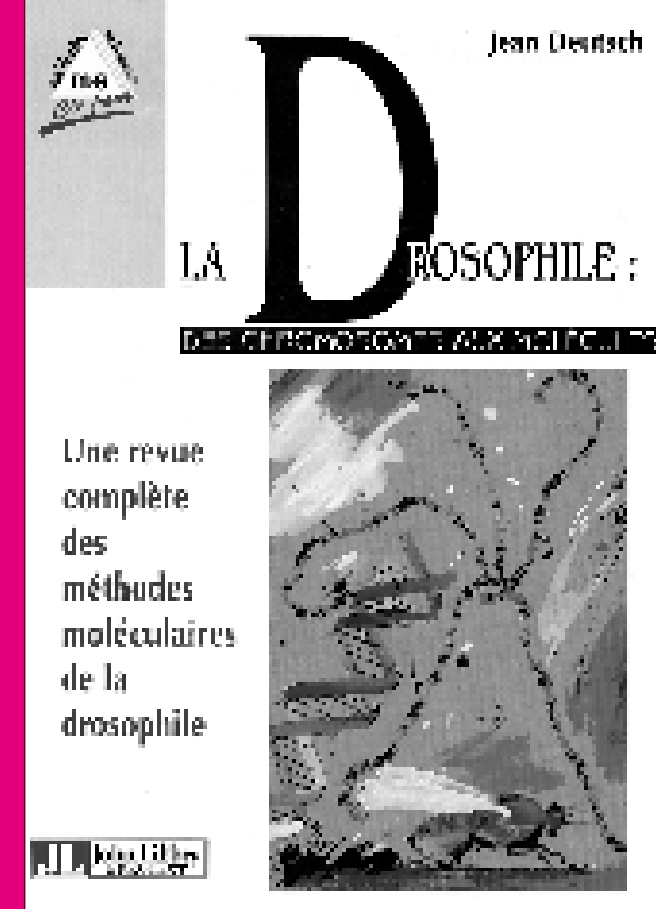

\section{BON DE COMMANDE}

A retourner aux : Éditions John Libbey Eurotext : 127, avenue de la République - 92120 Montrouge - FRANCE

\section{Tél.: 33 (1) 46.73.06.60-Fax: 33 (1) 40.84.09.99}

$\square$ Je souhaite recevoir LA DROSOPHILE

au prix de $145 \mathrm{FF}+25 \mathrm{FF}$ de frais de port, soit $\mathbf{1 7 0} \mathrm{FF}$

Paiement :

$\square$ par chèque à I'ordre de John Libbey Eurotext

$\square$ par carte bancaire :
O visa
Eurocard/Mastercard
American Express

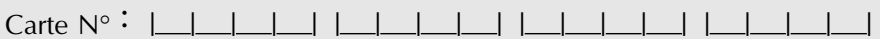

Collection :

Clé pour médecine/sciences

Un volume broché, 120 pages

ISBN : 2-7420-0062-3

Prix : 145 FF

\section{La Drosophile :} des chromosomes aux molécules Jean Deutsch

\author{
LA DROSOPHILE A L'HONNEUR \\ Christiane Nüsslein-Volhard \\ Edward B. Lewis \\ Éric Wieschaus
}

Prix Nobel de Médecine 1995 